\title{
Stratégies dénominatives en onomastique commerciale
}

\author{
Christine Fèvre-Pernet \\ Equipe de Recherche en Syntaxe et Sémantique (ERSS) \\ pernet@univ-tlse2.fr
}

\section{Introduction ${ }^{1}$}

Dans le domaine commercial le nom participe d'une stratégie communicationnelle qui doit mener à l'acte d'achat. Le nom doit avoir un fort potentiel de séduction et de conviction. Comment nomme-t-on dans une perspective commerciale?

On étudiera ici plus particulièrement les noms de produits ou les noms de branduit (c'est-à-dire des noms qui sont à la fois nom de marque et nom de produit, dans le cas où un produit unique est commercialisé au départ par l'entreprise (type COCA-COLA). La distinction nom de marque/nom de produit est importante pour l'étude de l'onomastique commerciale et elle a été faite ailleurs (Fèvre-Pernet \& Roché 2005). Dans une perspective de caractérisation de la construction du sens, les noms de produits constituent un bon terrain d'observation dans la mesure où le référent est « concret ». Les marques renvoient quant à elles, à des entités abstraites.

La littérature sur l'onomastique commerciale (parfois réduite à « nom de marque ») est relativement abondante. De nombreux ouvrages émanent des sphères du marketing. Les linguistes ont également travaillé sur le sujet (Praninskas 1968, Picone 1996, Platen 1997). Nous retiendrons des travaux des «markologues » (comme ils se définissent eux-mêmes) l'éclairage pragmatique qu'ils apportent en particulier sur le type de motivation qui préside à la création de ces noms (entre autres Bessis \& Bessis 1998). Les travaux des linguistes insistent sur l'aspect formel des créations publicitaires en lien le plus souvent avec le sémantisme des noms de marques mais sans systématiser l'étude de la construction du sens. Ce que nous proposons ici est de donner quelques résultats d'une analyse qui combine morphologie, sémantique et pragmatique (au sens de prise en compte du contexte).

\section{Des outils pour rendre compte de la construction des mots}

En nous appuyant sur les cadres d'analyse proposés par Roché (à paraître, en préparation a et b), nous abordons la formation des noms de produits en mettant en oeuvre une approche onomasiologique (schèmes de nomination) et une approche sémasiologique (opérations constructionnelles). La distinction entre dénominations endocentriques et exocentriques est également importante notamment pour l'étude des composés. L'analyse des productions issues de l'onomastique commerciale exige certains positionnements et certains choix qui passent par la prise en compte du contexte et de ses paramètres. Dans le cadre de cette étude, nous avons fait certains raccourcis mais le but était de disposer d'une grille d'analyse (à gros tamis) pour opérer par la suite un classement plus précis des productions rencontrées en onomastique commerciale.

\subsection{Schèmes de nomination et formats dénominatifs}

Michel Roché (à paraître), dans une approche qui envisage un traitement unifié des phénomènes de construction des mots, pointe l'importance de la prise en compte des schèmes de nomination conjointement à celle des procédés constructionnels. Bien souvent l'approche est unique : soit onomasiologique (et on est dans le cadre général de la sémantique référentielle), soit sémasiologique (et alors seules les opérations et règles de construction sont prises en compte). Or pour rendre compte des stratégies de nomination en onomastique commerciale, il est indispensable de croiser les deux approches. 
L'approche onomasiologique part de l'objet lui-même pour aller vers le mot. La question est alors celle $\mathrm{du}$ « schème de nomination » utilisé. Roché appelle ainsi la relation qui associe un mot construit, en tant que dénomination d'une classe d'objets ou de substances, de procès ou de qualités, à un autre mot du lexique. Nous reprenons dans ce qui suit les exemples de l'auteur. Un oiseau, par exemple, peut être dénommé d'après ses mœurs (grimpereau), son régime alimentaire (chardonneret), sa couleur (verdier) ou une autre caractéristique physique (gros-bec), etc. ; un instrument d'après le procès dans lequel il intervient (arrosoir, imprimante, calculatrice, lave-linge, machine à écrire...), d'après le nom de son inventeur ou promoteur (cardan, guillotine, diesel...), d'après une marque dominante (frigidaire, pédalo, karcher...) ; etc. L'étude du corpus de noms de produits révèle de la même façon certaines régularités, des modes de dénomination, des « schèmes de nomination » récurrents.

L'approche sémasiologique part du mot existant - la base - pour analyser les opérations constructionnelles qui aboutissent au mot construit. Selon son acception la plus courante, un lexème est caractérisé par (1) sa forme phonologique, (2) son sens lexical, (3) l'appartenance à une catégorie grammaticale. La construction d'un nouveau lexème comprend donc, potentiellement, une opération formelle, une opération sémantique, une opération catégorielle (mais l'une ou l'autre, voire deux d'entre elles peuvent ne pas être activées). Le mot construit résulte de la mise en œuvre de ces opérations et s'inscrit ainsi dans un certain format que nous appellerons ici « format dénominatif». Ce terme renvoie à un patron de formation identifié et spécifié en termes d'opérations constructionnelles et va nous permettre de classer les créations observées dans notre corpus.

L'opération formelle peut consister en l'ajout d'un affixe (préfixation, suffixation) ou d'un autre lexème (coalescence ${ }^{2}$ ); en la réduction ou le remaniement du matériau phonique (apocope, siglaison, formations à redoublement...). Elle n'est pas activée dans la conversion (chant(er) --> (le) chant, orange $\mathrm{N} \mathrm{-->}$ orange Adj) ou dans la dérivation sémantique (café 'boisson' --> café 'établissement', souris 'animal' --> souris 'accessoire d'ordinateur') (Roché à paraître).

L'opération sémantique peut consister en une simple altération de la capacité référentielle du lexème (restriction, par exemple, dans maison --> maisonnette) ou en un véritable transfert. Transfert de type processif, qui permet de nommer le procès à partir de l'un de ses participants (marteau--> marteler) ; transfert de type actanciel, du procès à un de ses participants (vol(er) --> voleur, arros(er) --> arrosoir) ou d'un participant à un autre (pétrole --> pétrolier ${ }^{3}$ ) ; transfert par contiguïté : métonymie (café 'boisson' --> café 'établissement'), méronymie (barbe --> barbu) ; transfert par analogie (souris 'animal' --> souris 'accessoire d'ordinateur', éléphant --> éléphantesque) ; etc. ${ }^{4}$ Sans affecter la capacité référentielle du lexème (dénotation), elle peut modifier son registre diaphasique - hypocoristiques à redoublement (fille --> fifille), abréviations (manifestation --> manif), suffixation substitutive (valise --> valoche), etc. - ou ses connotations.

L'opération catégorielle est une « recatégorisation », d'une catégorie majeure à une autre (meuble $\mathrm{N}$--> meubler $\mathrm{V}$, arroser $\mathrm{V}$--> arrosoir $\mathrm{N}$, orange $\mathrm{N} \mathrm{-->} \mathrm{orange} \mathrm{Adj,} \mathrm{etc.)} \mathrm{ou} \mathrm{d'une} \mathrm{sous-catégorie} \mathrm{à} \mathrm{une} \mathrm{autre}$ (Champagne Npr --> champagne Nc, cerise Nc --> Cerise Npr, etc.). Les noms de marques - en particulier les marques anciennes -- sont souvent obtenus par recatégorisation d'un anthroponyme (Nathan, Renault, Berchet...) $)^{5}$. La composante catégorielle n'est pas activée dans la dérivation évaluative (maison --> maisonnette, valise --> valoche).

Moyennant quelques aménagements, les mêmes outils (schèmes de nomination, formats dénominatifs résultant des opérations constructionnelles) sont utilisables pour analyser la formation de ces lexèmes particuliers que sont les noms de produits (et les noms de marques), les noms de produits étant à mi-chemin entre nom commun et nom propre. Le statut de ces noms a été largement discuté (entre autres, Siblot (1995), Fèvre-Pernet \& Roché (2005), Laurent (2006, 2007), Petit (2000, à paraître)).

\subsection{Dénominations endocentriques, dénominations exocentriques}

Dans l'analyse des noms de produits, nous utiliserons également la distinction traditionnelle entre dénominations endocentriques et dénominations exocentriques. On considère qu'on est en présence d'un 
$\mathrm{Npd}^{6}$ endocentrique lorsque le noyau sémantique est contenu dans le Npd, comme dans les composés CARREPONGE ${ }^{7}$, PUR SOUP', par exemple. Les Npd classés comme exocentriques sont ceux qui n'ont pas leur noyau sémantique à l'intérieur du composé. Ces mots sont en général formés par transfert sémantique. Par exemple : NUIT ENCHANTEE (mobile pour bébé). On peut étendre cette distinction aux noms formés par un autre procédé que la composition : SOLEILOO (hochet en forme de soleil), PYRAMIDE (jeu de société basé sur la forme et le principe de la pyramide). Cela revient à considérer que les noms exocentriques sont ceux qui sont construits par une opération sémantique de transfert (métonymie le plus souvent). La distinction endocentrique/exocentrique est importante car elle l'un des critères qui nous permet de comparer la création interdomaine en onomastique commerciale en termes de transparence du message délivré par le nom.

Cette distinction endocentrique/exocentrique est ramenée à la distinction transparent/opaque chez Ronneberger-Sibold (2006) qui s'appuie sur ce critère pour proposer une classification des mots-valises en fonction du degré de transparence conservé par les formants. Elle travaille à partir de deux corpus contrastés, l'un concernant des productions satiriques l'autre des noms de marques.

\subsection{Points de vue pour l'analyse : prise en compte du contexte}

Pour mener l'analyse, nous replaçons l'acte de nomination dans le contexte de la communication. Cette prise en compte de la situation de communication est donc un élément central pour l'analyse. Pour spécifier les paramètres de cette situation nous avons recours au concept de "genre de discours ». On peut envisager d'articuler la notion de genre plus traditionnellement convoquée pour les «textes »- aux systèmes dénominatifs.

Il semble en effet que les choix dénominatifs puissent être analysés en lien avec les outils utilisés pour l'analyse des genres du discours pour plusieurs raisons : (i) l'existence d'une sphère de communication spécifique $^{8}$ (commerciale); (ii) la présence de visées spécifiques (faire acheter) ; (iii) l'existence d'un destinateur et d'un destinataire clairement identifiés ; (iv) les choix linguistiques opérés compte tenu des trois premiers éléments et en fonction de modèles dénominatifs préexistants (ressources de la langue) ; (v) la relative stabilité du domaine nominatif concerné (alimentation, produits d'entretien, jouets...).

Cette mise en relation s'effectue grâce à la notion de schème de nomination. La visée de la communication conditionne les choix de ces schèmes tout comme elle conditionne les choix d'expression (à plusieurs paliers) pour le texte. On peut donc élargir à l'acte dénominatif ce que dit Bakhtine (1984) à propos des choix linguistiques opérés sous la contrainte du genre.

Le choix du nom est bien un acte à travers lequel s'exprime une visée particulière dans le cadre de la sphère commerciale. Dans l'univers commercial, la dénomination d'un produit destiné à la vente est étroitement liée à la visée prescriptive (faire acheter). Cette visée prescriptive est englobante et se distribue en trois "sous-visées »: une visée informative (faire savoir), une visée pathémique (faire ressentir) et une visée incitative (faire croire) (Charaudeau : 1995). La visée informative est réalisée par les schèmes de nomination descriptif et actanciel, la visée pathémique par le schème de nomination associatif et la visée incitative par le schème de nomination appellatif ( $\mathrm{cf} \S$ 4.2.).

De nombreux travaux portent sur l'identification des visées illocutoires dans le domaine publicitaire, on peut citer entre autres Adam \& Bonhomme 2005, Bonhomme 1997. Tout le monde s'accorde sur le «processus de positivisation ${ }^{9}$ sous-jacent qui s'applique à toute production publicitaire et donc au nom de marque et au nom de produit. 


\section{Quel corpus pour quelles analyses?}

\subsection{Des données contextualisées}

Des outils convoqués plus haut, découle la nécessité de garder un lien étroit avec le contexte général dans lequel s'opère le recueil des données. Ce lien au sens contextuel initial est aussi important pour les données morphologiques que pour les données textuelles, même si cela est moins communément admis dans le domaine de la morphologie (certains « corpus » sont encore des listes de mots « décontextualisés »).

Pour constituer notre corpus, nous avons donc dû garder (et collecter) des informations sur le produit nommé. Il s'agit de conserver le maximum d'informations sur ce produit afin d'établir le plus fidèlement possible de quel schème de nomination relève le $\mathrm{Npd}$.

Le premier corpus (corpus Galliot) a été constitué à partir d'un ouvrage paru dans les années 50 (Galliot : 1955). L'intérêt de cet ouvrage est de fournir des contextes explicatifs ${ }^{10}$ aux 500 noms collectés. Les analyses morphologiques sont un peu datées mais les informations sémantiques sont très fines et permettent de reconstituer le schème de nomination utilisé.

Le second corpus (corpus contemporain) est un corpus contemporain qui s'étend sur la période 1980-2006). Il a été constitué à partir du corpus existant de Jacquemin (1984) et d'un relevé effectué par nos soins à partir de sites marchands sur le web (Telemarket...), de prospectus ou de catalogues commerciaux (900 noms). Le plus souvent la photographie de l'emballage a été enregistrée ou consultée de façon à obtenir des informations au moment de l'analyse du nom, le petit texte ou le sous-titre explicatif figurant sous les textes (site web) a également été consulté.

\subsection{Variations par sous-domaines, variations par époque}

La variation a été observée selon les deux composantes détaillées plus haut : schèmes de nomination et formats dénominatifs.

Nous avons observé la variation liée aux différents produits nommés. Ces produits s'inscrivent dans des sous-domaines (voire sous-sous domaines) de l'onomastique commerciale: produits d'entretien, alimentation (fromages, biscuits, bonbons, boissons...), produits manufacturés (jouets, électroménager, sous-vêtements...), etc. On ne nomme pas de la même façon un paquet de biscuits, un parfum ou un aspirateur. L'objectif est d'observer l'influence du sous-domaine d'appartenance sur le choix d'un schème de nomination et d'un format dénominatif.

Il est parfois difficile de dater avec précision la première apparition d'un nom de marque et plus encore d'un nom de produit. Nous avons réalisé une vérification rapide sur internet à partir du corpus Galliot pour voir si certains noms existaient toujours. Nous avons soustrait ces noms du corpus contemporain. Il reste que nous n'avons pu matériellement vérifier tous les noms du corpus contemporain : si certains noms sont apparus dans les années 70, ils figurent quand même dans ce corpus.

Pour observer la variation diachronique il a fallu également partitionner le corpus et comparer par sous-domaines à l'époque de Galliot et à l'époque contemporaine : les noms de boissons, les noms de biscuits, les noms de produits d'entretien, les noms de sous-vêtements, de produits de parfumerie... Mais les types de référents ont évidemment beaucoup changé. Certaines classes référentielles ont disparu. L'analyse quantitative que nous projetions s'est révélée assez difficile à mettre en œuvre du fait de la difficulté à obtenir un réel parallélisme entre les époques. Des observations ont pu néanmoins être réalisées sur quelques domaines. Plus qu'une mesure de la variation c'est la nature de la variation qui nous intéresse. Plus particulièrement, cela peut nous éclairer sur la stabilité relative des schèmes et formats de nomination utilisés. 


\section{Caractériser un « genre dénominatif »}

Chaque nom créé en onomastique commerciale résulte de l'application d'un format dénominatif et d'un schème de nomination. En d'autres termes, la création résulte de l'application d'un format et d'un schème à un référent. Le schème de nomination choisi renseigne sur la stratégie de nomination employée. On présentera les résultats obtenus selon les deux approches évoquées plus haut (§ 2.1.): tout d'abord les formats dénominatifs - les mots construits et répertoriés selon les procédés utilisés en morphologie constructionnelle/lexicale. Ensuite nous proposerons une typologie empirique des schèmes de nomination - résultat de l'observation des données de notre corpus.

\subsection{Formats dénominatifs en onomastique commerciale}

Les noms de l'onomastique commerciale, que l'on considère comme des mots construits, sont étudiés selon leur aspect constructionnel et donc selon les opérations formelles, catégorielles et sémantiques dont ils résultent. Dans ses propositions, Roché s'appuie sur les trois composantes définies par Danielle Corbin (1987, 1991) mais sans les faire dépendre d'une règle. Tout en cherchant à dégager des régularités de fonctionnement, nous ne soumettons pas notre corpus à une analyse mettant en œuvre des règles strictes à visée généralisante. Il nous importe de rendre compte des phénomènes rencontrés dans l'ensemble du corpus, l'onomastique commerciale présentant des éléments très hétérogènes. Pour chacun des deux grands procédés - dérivation et composition, nous considérons qu'il existe un format prototypique (dérivation prototypique, composition prototypique) à partir duquel nous pouvons organiser la description. Nous considérons qu'il existe un continuum (du plus ou moins prototypique) et pouvons ainsi regrouper sous un même format dénominatif des créations qui pour d'autres ne seraient explicables que par un phénomène d'analogie ou qui même seraient rejetées hors du domaine de la morphologie.

Dans ce corpus, tous les procédés de formation classiques sont utilisés, de la composition (COCOTTE-MINUTE, DECAP FOUR) à la dérivation (suffixale : SOUPLINE ; préfixale : MINIZZA ; conversion : RELAX), même si des libertés sont prises par rapport à certains d'entre eux. On établira quels sont les procédés les plus employés par domaine et par époque en croisant les critères.

\subsubsection{Dérivation}

\section{Suffixation}

Dans le but de conserver une certaine rentabilité explicative, on est amené à regrouper sous un même procédé - ici la suffixation - des formations non prototypiques. Certaines des créations classées ici ne répondent pas totalement au cahier des charges de ce procédé. La base peut ne pas être celle attendue pour le type de dérivé concerné et le «suffixe » peut présenter une certaine instabilité sémantique, même si en général on constate une certaine stabilité en matière de connotation. On accorde le statut de «suffixe» à certains formants qui ailleurs ne le sont pas. Dans les énumérations figurant ci- après, peuvent se côtoyer aussi bien des suffixes grammaticalisés et donc dotés d'un statut de suffixe dans la langue générale que des suffixoïdes ou pseudo-suffixes.

Dans le corpus contemporain la dérivation suffixale représente environ $32 \%$ des formats dénominatifs du corpus. C'est dans le domaine alimentaire (hors boissons) qu'elle est le plus représentée. Dans le corpus Galliot, elle tient une place importante $(20 \%)$. Nous avons observé une évolution d'une partie du matériel suffixal.

- Certains « suffixes » utilisés dans les années 20-50 ont presque complètement disparu. Ils sont absents du corpus contemporain. Par ordre de productivité décroissante (à l'époque et dans le corpus Galliot) : -o,-ö̈d, -bus, -ose, -ite, -ode.

- Certains ont perduré : - $a$, -ia, -ea, -ine, -ette, -ic, - $(e) x,-o r$, -os, -um, -on (toujours par ordre de productivité décroissante de gauche à droite). $-a$, -ia avaient une forte productivité et l'ont gardée en particulier dans les domaines où la cible est féminine (diététique, hygiène-parfumerie). 
- De nouveaux «suffixes » sont apparus dans le domaine de l'onomastique commerciale : -ou, -elle, -esse, -ade, -issime, -el, -ys, -is, -ance, -ière. Et également certains d'origine étrangère -y/ies, -ina, -ita(o), -etti, -ola, -ello, -er, -en, -als.

\section{Préfixation}

Comme pour la suffixation, on adopte une acception assez large du procédé de préfixation. On peut même faire entrer dans la catégorie des préfixes des formants qui sont ailleurs considérés comme des adjectifs. On considère que la stabilité de l'instruction sémantique qu'ils véhiculent peut leur conférer ce statut.

La préfixation est surtout productive dans le domaine des cosmétiques. Les préfixes hyper-, super-, ultra-, $b i-$, tri-... ont un contenu sémantique évaluatif dans une stratégie de communication qui cherche à persuader de l'efficacité des produits vendus. Un certain nombre de préfixes entre également dans la formation des noms de jouets, qu'ils soient également intensifs (super-, maxi-, méga-) ou qu'ils servent à spécifier la taille réduite du jouet (mini-, baby-...). On peut trouver BABYQUAD ou MINIQUAD pour désigner un quad de taille réduite : baby- est l'équivalent de mini- pour le référent étudié. La frontière avec la composition est alors assez floue mais au regard de la fréquence d'emploi de ces formants comme éléments présentant les mêmes propriétés qu'un préfixe, on classera les noms créés dans le format dénominatif « préfixation ».

En matière de variation, les préfixes les plus productifs chez Galliot sont extra-, néo- super-, ultra-... ; celui-ci note que super- a supplanté hyper-. Dans le domaine des cosmétiques aujourd'hui c'est plutôt le contraire : hyper- est plus productif que super-.

\section{Dérivation non affixale}

On classe dans la dérivation non affixale les noms construits par recatégorisation : l'opération catégorielle est l'opération principale. Il peut s'agir d'une recatégorisation qui affecte des catégories générales (adjectif en nom) ou des sous-catégories (nom commun en nom propre par exemple, nom propre en Npd).

La recatégorisation est très utilisée dans la formation des noms de parfums (CALECHE, POISON, PARIS...), dans les noms de boissons (MALIBU, SOHO...). La recatégorisation est associée à une opération sémantique. Le corpus Galliot privilégie la recatégorisation de Npr en Npd (THESEE, RONCEVO, ATTILA, ROUlETABILlE...). Ces noms propres sont souvent tirés de la mythologie ou encore de l'histoire événementielle ou littéraire mais tous ces noms sont issus du stock lexical partagé par le groupe à une époque donnée.

\subsubsection{Composition}

On regroupe sous le terme de «composé » des constructions allant du composé classique NN (COTON-TIGE) à une séquence qui résulte du figement d'un syntagme ou d'un fragment d'énoncé (par exemple relatives KIRECUR, KY-NET-TOY) et dont la construction avant figement relève de la syntaxe libre. On ne détaille pas ici toute la gamme des composés, on se limite à ces quatre types :

- composition traditionnelle, de type déterminé-déterminant, VN (DECAP FOUR, BOUF'ROUILLE), NN (VERNICIRE, COCOTTE-MINUTE), NAdj ou AdjN (GRATTON-LAVEUR, PUR SOUP') ;

- composition allogène, qui inverse l'ordre des formants, savante ou pseudo-savante (TAUPICIDE, DIGICODE), anglo-saxonne ou angloïde (CAMPING-GAZ), mixte (QUICK-LAIT), ou combinant des éléments français (APERICUBES, CROUSTIFRITES), mais toujours dans l'ordre déterminant/déterminé;

- composition syntagmatique (CARRE FRAIS), avec souvent des modifications orthographiques (PATAFIX [= pâte à fixer]).

- figement d'un syntagme complet (LE P'TIT GRILLE, MONSIEUR PROPRE, LA FASCINANTE). 
Au procédé de composition proprement dit s'ajoute celui de la troncation et de la superposition qui peut intervenir à des degrés différents dans la création de mots-valises ou pseudo-mots valises ${ }^{11}$. Nous ne les détaillerons pas ici.

Tous les domaines font appel au procédé de la composition notamment au travers des mots-valises qui permettent de condenser formellement le message à délivrer. Mais les composés peuvent également être de véritables syntagmes quand il s'agit de singulariser le produit (syntagmes définis).

Les figements de syntagmes ou de fragments d'énoncés sont beaucoup plus présents dans le corpus Galliot (MATEINTUR, LA CRESSONNEE, FOURBITOU, JASPIR, RAZVITE) que dans le corpus contemporain. Les mots-valises sont présents également mais en moins grand nombre et ne subissent pas de troncations importantes (LAVABLAINE).

Globalement, l'utilisation des différents procédés de construction n'est pas très différente d'une époque à l'autre. On note cependant une augmentation notable de la part des dérivés (32\% pour le corpus contemporain contre $20 \%$ chez Galliot).

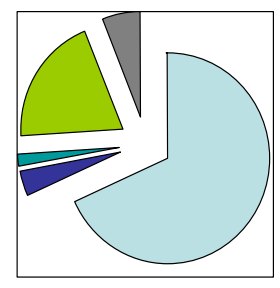

\begin{tabular}{|ll|}
\hline$\square$ Composé & $\square$ voc. Général \\
$\square$ Npr & $\square$ dérivé \\
$\square$ autre & \\
\hline
\end{tabular}

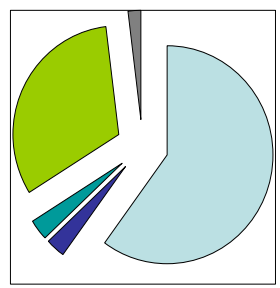

Dans le paysage de l'onomastique commerciale, les noms créés peuvent être très différents d'un domaine à l'autre. Les noms de parfum sont très souvent construits par recatégorisation d'un mot du vocabulaire général alors que les noms de produits diététiques sont plutôt construits par dérivation affixale. En fonction du domaine auquel appartient le référent, les formats dénominatifs sélectionnés varient. Varient également les schèmes de nomination mobilisés dans le processus de création du nom.

\subsection{Les schèmes de nomination en onomastique commerciale}

L'analyse du corpus permet de faire apparaître des récurrences en matière de schèmes de nomination. Les référents qui sont nommés sont des produits (alimentaires, d'entretien...) ou des objets manufacturés, nous n'avons retenu dans notre corpus ni noms de magasins ni de services (de type bancaire par exemple). A ce stade de l'étude, nous avons tenté de regrouper les observations réalisées en quatre types de schèmes: schèmes descriptifs, schèmes actanciels, schèmes associatifs, schèmes appellatifs. Ces schèmes principaux ou «macro-schèmes » peuvent chacun se subdiviser, le cas échéant, en catégories plus spécifiques.

\subsubsection{Schème descriptif}

Le mode de dénomination s'appuie sur une caractéristique du produit. Il peut s'agir : 
- d'une caractéristique perceptuelle : couleur, lumière (LUMINOU jouet luminescent) forme du produit (CARREPONGE), toucher (KANDOO, savon), sensation (GRANOLA)...

- d'un élément qui entre dans la composition du produit : BANANIA (poudre pour petit déjeuner avec du cacao et de la farine de banane), POUDRESOIE (poudre cosmétique), ORANGINA, MENTOS...

- du mode de fonctionnement de l'objet manufacturé : THERMOR (une cuisinière), GETIEN (soutien-gorge)...

- du destinataire du produit : KINDER, KIDIBOO, KIDEX, FAMILIA, X-MEN...

La dénomination peut également s'appuyer sur le nom du produit générique. Par exemple MONSAVON nom d'un savon lancé par la marque L'Oréal en 1930 - permet de catégoriser instantanément le produit/référent. En ce sens la dénomination est transparente et donc «descriptive » globalement. Ces dénominations correspondent aux mots construits endocentriques.

\subsubsection{Schème actanciel}

Il s'agit d'un schème (que l'on aurait pu nommer également proto-agentif ${ }^{12}$ ) qui fait appel à la notion de rôles sémantiques directement ou indirectement.

- Le produit est nommé d'après l'action qu'il réalise (relation Agent) : le nom est donné d'après l'action que permet d'effectuer le produit (JASPIR pour un aspirateur, KIRECUR, BEBE MOUILLEUR (un poupon qui fait pipi), BATON SAUTEUR (jouet qui permet de faire des sauts).

- Le produit est nommé d'après le patient auquel il applique son action : FURNEX (produit pour nettoyer les fours).

- Le produit est nommé d'après le résultat qu'il permet d'obtenir : LE POT-AU-FEU (une cuisinière), BOSSEIN (soutien-gorge), MEANEUF, BRILLFORT (produits d'entretien). Les trois derniers combinent au schème résultat un schème axiologique dans la mesure où il y a laudation du produit. On peut considérer que le produit est nommé d'après le bénéfice qu'il apporte au consommateur : NUIT CALME (tisane), NUIT ENCHANTEE (mobile qui endort bébé), KIN'KAS'PAH (lacets).

\subsubsection{Schème associatif}

Avec le schème associatif on bascule du dénotatif au connotatif. On s'éloigne de plus en plus du référent lui-même pour en construire une image singulière. Il n'est plus question de catégoriser mais de créer un univers autour du produit, en adoptant une stratégie argumentative qui inscrit en creux les valeurs des consommateurs cibles pour mieux emporter leur adhésion. L'acte de nomination a ici explicitement une dimension argumentative. On distinguera des stratégies énonciatives, des stratégies axiologiques (laudation du produit ou valorisation de la marque), des stratégies affectives (hypocoristiques), des stratégies interdiscursives (puiser dans le « déjà dit» lexical ou discursif), des stratégies créatrices d'univers (un ailleurs géographique ou diaphasique connoté).

Parmi les stratégies énonciatives, l'utilisation du possessif (en général la première personne du singulier) est assez fréquente. Dans le domaine du jouet la relation de l'enfant au jouet est ainsi mise en avant (MON PREMIER BALADEUR, MON MIROIR MAGIQUE, MON BEBE NURSE...). L'utilisation des adjectifs possessifs dans les noms de jouets trouve une explication à la fois dans la psychologie enfantine et dans celle de l'acheteur. Brée (1993) étudie la psychologie de l'enfant dans le cadre du marketing note qu'

une caractéristique importante de la pensée enfantine est sans doute son "égocentrisme". Il est dans un état d'immédiatisme égocentrique" (la conscience ne connaît que des objets singuliers sans interaction les uns avec les autres). Jusqu'à 6-7 ans, il ne fait que partiellement la différence entre lui et son environnement. (p. 26) 
Il s'avère justement que les jouets désignés par ces tournures possessives sont très souvent des jouets pour petits (moins de 6/7ans). Les objets qui entourent l'enfant se définissent donc par rapport à lui mais font aussi partie de lui. Dans le langage courant, un tout-petit associe identification à appropriation : quant il dit en voyant un objet «à moi, ça », l'appropriation précède même l'identification. C'est ce trait que peuvent traduire ces formes possessives.

Mais les composés syntagmatiques avec possessifs sont présents dans d'autres domaines (MATEINTUR, MONPINARD...). Everaert-Desmedt (1984) avance une explication en lien avec la sphère de l'échange. Le discours publicitaire montre souvent le consommateur conjoint au produit. Le fait d'appeler un savon MONSAVON, c'est déjà un pas vers l'acte d'achat, puisqu'il y a une appropriation anticipée par le langage. Adam \& Bonhomme (2005: 49-50) pointent le rôle des pronoms de première personne dans le texte publicitaire. En venant occuper la place énonciative du «je», le consommateur cible est immergé dans un univers euphorique partagé. Il reste que cette analyse concerne davantage le discours sur le produit que le rapport direct au produit.

Le recours à des évaluatifs sous quelque forme que ce soit est une stratégie dénominative de type axiologique : LE VRAI TELECRAN (une ardoise magique pour enfants), ÇA-VA-SEUL (produit d'entretien), LE REVE (une cuisinière), tout un paradigme de noms de produits en -lux (FRIGELUX, ELECTROLUX, SPORTLUX...) la finale -lux pouvant être interprétée comme le formant luxe (troncation $\mathrm{du}$ 《e » graphique). Et encore LA PARFAITE (lavette), LA FASCINANTE (laine LE LABOUREUR), INDESTRUCTOR (porte-habit), CHARMEREINE STAR (une gaine). De type axiologique également le recours à des mots ou terminaisons grecs ou latins qui «font sérieux» autant que savant (SVELTOR, HUMECTOR, MINTHOS, KRONOS, PHAGOS).

La stratégie peut être également de créer l'illusion d'une relation affectueuse consommateur/objet. Dans GRINDOLINE, CROQUINE, BAGUETTINE, CHIPTELLE, INFUSELLE, FRITELLES, TOASTINETTE, les suffixes apportent une dimension hypocoristique. Même chose pour le suffixe anglais -y (SPIKY pour un jouet-perroquet parlant, SUPER FRAISY), ou pour le suffixe -ou (variante graphique -oo) (KIDIBOO).

L'empathie avec le consommateur est très souvent travaillée dans la sphère commerciale par le biais de stratégies interdiscursives : il s'agit de s'assurer de sa complicité en lui offrant de décrypter une dénomination qui fait appel à un savoir partagé. La notion de savoir partagé est une notion mobilisée dans l'étude de l'argumentation ordinaire. Le produit peut recevoir un nom qui est déjà en mémoire dans le lexique d'une façon particulière : LE DIABLE ROUGE (une cuisinière et, sans le déterminant défini, aussi un aspirateur de la marque VIBELL) ROULETABILLE (un stylo), RONCEVO (klaxon), JERICHO (klaxon), ATTILA (raticide). Pour construire le Npd on puise dans le « déjà-dit » lexical et discursif. Le donneur de nom compte sur l'activation de la mémoire de ces noms et sur le choc (ludique) créé par l'association des deux référents. L'effet de connivence, s'il a correctement fonctionné, entraîne une meilleure mémorisation du nom et un positionnement favorable par rapport à la marque ou au produit.

Enfin pour terminer cet inventaire des schèmes associatifs on peut également évoquer les stratégies créatrices d'univers (un ailleurs géographique ou diaphasique connoté). Cette stratégie du déplacement se retrouve par exemple dans les noms de boissons (SOHO, MALIBU...) qui utilisent des lieux géographiques à fort pouvoir évocateur. Outre l'exotisme, l'effet «terroir » peut être recherché avec par exemple des finales connotées (COCHONNOU) mais il s'agit alors d'un autre type de produit. D'autres modèles de vie à pouvoir valorisant peuvent être convoqués et notamment l'anglais : KISS (bas, rouge à lèvre), GAINEWELL dans le corpus Galliot. L'espagnol et l'italien sont également présents au travers de certains suffixes. Les « suffixes féminins »-esse, -essia, - $a$, -ia contribuent également à créer un univers typiquement féminin prenant ainsi en compte les destinataires des produits vendus.

\subsubsection{Schème appellatif}

$\mathrm{Ce}$ dernier schème semble correspondre à une volonté d'individualisation et même d'anthropomorphisation du produit puisqu'il s'agit de baptiser le produit de la même façon qu'une personne. Certains objets se prêtent naturellement à ce type de dénomination appellative comme les 
poupées ou peluches qui peuvent être directement nommées par le fabricant. On peut faire appel à un prénom déjà présent dans le stock lexical ou en créer un de toutes pièces. En ce qui concerne d'autres produits c'est le discours sur le produit, les visuels accompagnant sa présentation au public qui vont asseoir sa dimension anthropomorphique. La campagne publicitaire sur la R5 (une voiture dotée de deux yeux et d'une bouche, qui en plus parlait...) marquait dans les années 70 le début de la volonté d'anthropomorphisation qui s'est ensuite matérialisée par une stratégie désignative qui consiste à donner aux voitures des noms de « femme » (MEGANE, SAFRANE...).

De la même manière que pour les «formats dénominatifs », on peut observer la productivité de certains schèmes selon les domaines ou sous-domaines. Les boissons alcoolisées utilisent le schème associatif créateur d'univers (SOHO, MALIBU...). Les produits alimentaires restent attachés le plus souvent à un schème descriptif ce qui donne une certaine transparence au nom de produit.

Selon l'époque pour un même domaine le schème dominant peut changer: par exemple en ce qui concerne les produits manufacturés comme les sous-vêtements féminins on passe d'un schème descriptif ou actanciel (GETIEN, BOSSEIN) à un schème associatif créateur d'univers et axiologique (BILLETS DOUX, PASSIONATA, AUBADE, DESSOUS CHERI, NYMPHEA, RAVAGE...).

\section{Bilan et perspectives}

Les productions de l'onomastique commerciale présentent un certain degré d'hétérogénéité. Mais les analyses réalisées nous permettent d'établir un premier bilan descriptif. Tout d'abord en ce qui concerne les formats dénominatifs, la composition est le procédé de formation privilégié, mais tous les autres sont également utilisés. Ils le sont, le plus souvent, conformément aux procédés de la morphologie constructionnelle même s'ils s'éloignent du prototype. Les écarts sont souvent liés à la tendance à tronquer les formants, en particulier dans les composés (ce qui peut poser des problèmes quant à l'identification catégorielle du formant). La préfixation présente un fonctionnement assez proche de ce qu'elle est ailleurs, mais la suffixation prend des libertés avec l'instruction sémantique ou catégorielle attachée aux suffixes, ou avec les suffixes eux-mêmes (qui souvent n'en sont pas). La composition présente un visage très diversifié à cause de la forte présence de l'anglais, dans le choix des formants mais aussi dans celui des structures (ordre DaDé vs DéDa). En ce qui concerne les schèmes de nomination, les grandes lignes semblent avoir été repérées, en lien avec la dimension argumentative de la situation de communication. Il reste à affiner les catégories mises au jour pour établir une véritable typologie des schèmes de nomination utilisés en onomastique commerciale. Nous pouvons également envisager, une fois cette typologie stabilisée, d'interroger la notion dans le champ du « lexique ordinaire».

La notion de «genre dénominatif » mérite également d'être interrogée de plus près. Elle permet en effet de circonscrire les éléments pragmatiques qui entrent en jeu dans le processus de dénomination. La prise en compte de la sphère de communication dans laquelle a lieu l'activité de nomination a une importance dans la mesure où la visée de la communication a une influence certaine sur le choix de schèmes de nomination. L'opération de construction du sens ne peut être observée qu'à la condition de connaître les acteurs de la communication (directs et indirects), le support de la communication, les arrière-plans de l'époque en matière de savoir partagé. Le facteur dialogique ou l'intertextualité du lexique entre également en ligne de compte : un mot renvoie forcément à un mot antérieur et à un mode de construction déjà présent dans le lexique. Des pistes d'analyse peuvent être activées ou non en fonction du degré de saillance de ces divers éléments. Comme dans le discours, le sens n'est pas stabilisé dans le lexique : un mot dénote mais en fonction de sa circulation dans différents discours le mot peut se charger de connotations ou d'une nouvelle dénotation.

Enfin, une telle grille d'analyse peut être utile pour l'étude d'autres paradigmes dénominatifs dans la sphère des noms propres : noms de tableaux, noms de bateaux, noms de villas, noms de groupes de musique, etc. Elle présente l'intérêt de spécifier l'«air de famille» du paradigme en termes de formats et de schèmes dénominatifs. 


\section{Références bibliographiques}

Adam, J.-M. \& Bonhomme, M. (2005) L'argumentation publicitaire, Rhétorique de l'éloge et de la persuasion. Paris, Nathan

Bakhtine, M. (1984). Esthétique de la création verbale. Paris, Gallimard.

Bessis, M. \& Bessis, P. (1998). Les noms qui gagnent, l'alchimie des noms irrésistibles. Paris, LPM.

Bonhomme, M. (2002). "La publicité comme laboratoire du français actuel". L'Information Grammaticale 94, pp. 33-38.

Charaudeau, P. (1995). "Le dialogue dans un modèle de discours". Cahiers de linguistique française 17: 141-178.

Corbin, D. (1987). Morphologie dérivationnelle et structuration du lexique. Tübingen, Max Niemeyer Verlag, Villeneuve d'Ascq, P.U.L.

Corbin, D. (1991). "La formation des mots : structures et interprétations", Lexique 10, pp. 7-30.

Degauquier, C. (1994). "Etudes terminologiques et linguistiques : poétique contre peau étique - le lexique des cosmétiques". META 39 (3), pp. 465-470.

Galliot, M. (1955). Essai sur la langue de la réclame contemporaine. Toulouse, Privat Edouard.

Laurent, B. (2007). « Noms de marque, noms de produit : analyse d'une exemplarité de la créativité lexicale », Neologica 1, J. Humbley et J.-F. Sablayrolles (éd.)

Laurent, B. (2006) « Nom de marque, nom de produit. Production de sens en discours publicitaire ». Thèse de Doctorat, Université de Montpellier 3.

Petit, G. (2000). "Un hybride sémiotique : le nom déposé". Lingvistica Investigationes, 23, 1, pp. 161-192.

Petit, G. (à paraître). "Le nom de marque déposée : nom propre, nom commun et terme". Journée Conscila, mars 2003.

Picone, M. D. (1996). Anglicisms, neologisms and dynamic French. Amsterdam/Philadelphia, John Benjamins Publishing Company.

Platen, C. (1997). Okonymie : zur Produktnamen-Linguistik im Europäischen Binnenmarkt. Tübingen, M. Niemeyer.

Praninskas, J. (1968). Trade Name Creation. La Haye/ Paris, Mouton.

Roché, M., en préparation, a. "Quelle morphologie ?", in M. Roché, G. Boyé, H. Giraudo, N. Ha-thout, S. Lignon, F. Montermini, M. Plénat, Des unités morphologiques au lexique.

Roché, M., en préparation, b. Propositions en morphologie lexicale, Carnets de grammaire, Toulouse, CLLE-ERSS (Carnets de grammaire).

Roché, M. (à paraître). Pour une morphologie lexicale, Actes du colloque ????

Ronneberger-Sibold, E. (1999). "La création de connotations à valeur affective dans les noms de marques", SILEXICALES 2, Toulouse, pp. 219-227.

Ronneberger-Sibold, E. (2006). "Lexical Blends: Functionally Tuning the Transparency of Complex Words", Folia Linguistica XL/1-2, pp. 155-181.

Siblot, P. (1995), "Noms et image de marque : de la construction du sens dans les noms propres". In M. Noailly (éd.), Nom propre et nomination, Actes du Colloque de Brest, 21-24 avril 1994, pp. 147-160.

\footnotetext{
${ }^{1}$ Je remercie mes deux relecteurs anonymes qui, par leurs remarques et conseils, m'ont aidée à améliorer cet article.
} 
${ }^{2}$ Ce terme est proposé par Michel Roché (à paraître) pour remplacer «composition » qui a le désavantage de désigner à la fois l'opération formelle (ajout d'un lexème) et le procédé dans son ensemble (coalescence serait alors sur le même plan qu'affixation).

${ }^{3}$ Où pétrole est proto-patient et pétrolier proto-agent (Roché utilise la classification de Dowty, D. (1991). "Thematic Proto-roles and Argument Selection". Language in Society 67(3), pp. 547-619.

${ }^{4}$ Ces opérations de dérivation sémantique (métaphore, métonymie, etc.) sont souvent écartées, à tort, du domaine de la morphologie.

5 Cette «recatégorisation» suppose que l'on considère l'existence de «sous-catégories» du nom propre (anthroponymes, toponymes, noms de marques (ou « ökonyme » selon Platen (1997)...)

${ }^{6}$ Nom de produit sera abrégé en Npd et nom de marque en Nmq.

${ }^{7}$ Les noms de marques et les noms de produits sont notés en petites capitales.

${ }^{8}$ Qui définit l'ancrage social et institutionnel.

${ }^{9}$ Le terme est d'Everaert-Desmedt (Adam \& Bonhomme $2005: 58$ ).

${ }^{10}$ Informations portant sur la nature du référent, sur le destinataire du produit, sur le type de société commercialisant le produit...et surtout sur la motivation qui préside à la création du nom.

${ }^{11}$ Mots-valises qui ne sont pas conformes au mot-valise prototypique.

${ }^{12}$ Cf Dowty, D. (1991). 